International Journal of Applied Mathematics

Volume 34 No. 2 2021, 237-248

ISSN: 1311-1728 (printed version); ISSN: 1314-8060 (on-line version)

doi: http://dx.doi.org/10.12732/ijam.v34i2.2

\title{
SOLUTION OF TWO-PHASE CYLINDRICAL DIRECT STEFAN PROBLEM BY USING SPECIAL FUNCTIONS IN ELECTRICAL CONTACT PROCESSES
}

\author{
Stanislav N. Kharin ${ }^{1,2}$, Targyn A. Nauryz ${ }^{1,2,3,4} \S$ \\ ${ }^{1}$ Institute of Mathematics and Mathematical Modeling \\ Almaty - A26G7T4, KAZAKHSTAN \\ ${ }^{2}$ Kazakh British Technical University \\ Almaty - A05H1T2, KAZAKHSTAN \\ ${ }^{3}$ Al-Farabi Kazakh National University \\ Almaty - A15E3B4, KAZAKHSTAN \\ ${ }^{4}$ Satbayev University \\ Almaty - A15P4X4, KAZAKHSTAN
}

\begin{abstract}
In this work two-phase Stefan problem for the cylindrical heat equation is considered. One of the phase turns to zero at an initial time. In this case, it is difficult to solve it by radial heat polynomials because the equations are singular. The solution is represented in linear combination series of special functions, the Laguerre polynomial and confluent hypergeometric function. The free boundary is known. The undetermined coefficients of the heat in two phases and the heat flux are found. The convergence of special functions series is proved.
\end{abstract}

AMS Subject Classification: 35K05, 80A22

Key Words: confluent hypergeometric function; Laguerre polynomial; Faa-di Bruno formula; Stefan problem

\section{Introduction}

The cylindrical heat equation is important in mathematical modeling of heat transfer in bodies which cylindrical domain. The solution, in other words is

Received: November 17, $2020 \quad$ (C) 2021 Academic Publications

$\S$ Correspondence author 
a temperature distribution, in such model can take form of series of a special function (Laguerre polynomials). In the development of heat processes models, the partial differential equations play necessary role (see [1], [3]). The theory of free boundaries made tremendous progress in the last half century. We refer to Chen et al. [5] and Friedman [6], [7] in which the realization of some models can be expressed as free boundary problems are given. In the process of heat arcing, the phase transformation takes place. Therefore, we consider Stefantype problems [8], [9]. Present study is devoted to such Stefan problems in which temperature functions are considered in forms of special functions with unknown coefficients that have close link introduced by Rosenbloom and Widder [10]. To represent the solution form of the problem we consider the following equation

$$
x \frac{d^{2} \phi}{d x^{2}}+\left(\frac{\nu+1}{2}-x\right) \frac{d \phi}{d x}+\frac{\beta}{2} \phi=0, \quad \nu=0, \quad-\infty<\beta<\infty .
$$

It is well known that this equation has two linearly independent solutions

$$
\begin{aligned}
& \phi_{1}(x)=\Phi\left(-\frac{\beta}{2}, \frac{\nu+1}{2} ; x\right), \\
& \phi_{2}(x)=x^{\frac{1-\nu}{2}} \Phi\left(\frac{1-\beta-\nu}{2}, \frac{3-\nu}{2} ; x\right),
\end{aligned}
$$

where $\Phi(a, b ; x)$ is the confluent (degenerate) hypergeometric function. Setting $T(z)=\phi(x)$, where $x=-z^{2}$, one can find that $T(z)$ satisfies the equation

$$
\frac{d^{2} T}{d z^{2}}+\left(\frac{\nu}{z}+2 z\right) \frac{d T}{d z}-2 \beta T(z)=0
$$

Using this equation one can check up that the function

$$
\theta(z, t)=(2 a \sqrt{t})^{\beta} T\left(\frac{z}{2 a \sqrt{t}}\right)
$$

satisfies the equation

$$
\frac{\partial \theta}{\partial t}=a^{2}\left(\frac{\partial^{2} \theta}{\partial z^{2}}+\frac{\nu}{z} \frac{\partial \theta}{\partial z}\right)
$$

Hence, the functions

$$
\begin{aligned}
& S_{\beta, \nu}^{(1)}(z, t)=(2 a \sqrt{t})^{\beta} \Phi\left(-\frac{\beta}{2}, \frac{\nu+1}{2} ;-\frac{z^{2}}{4 a^{2} t}\right), \\
& S_{\beta, \nu}^{(2)}(z, t)=(2 a \sqrt{t})^{\beta}\left(\frac{z^{2}}{4 a^{2} t}\right)^{\frac{1-\nu}{2}} \Phi\left(-\frac{\beta}{2}, \frac{\nu+1}{2} ;-\frac{z^{2}}{4 a^{2} t}\right)
\end{aligned}
$$


satisfy the equation (3). If $\beta$ is an even integer, $\beta=2 n$, the function $S_{\beta, \nu}(z, t)$ can be expressed in terms of the generalized Laguerre polynomials

$$
\begin{gathered}
S_{2 n, \nu}^{(1)}(z, t)=\left(4 a^{2} t\right)^{n} \Phi\left(-n, \mu ;-\frac{z^{2}}{4 a^{2} t}\right) \\
=\frac{n ! \Gamma(\mu)}{\Gamma(\mu+n)}\left(4 a^{2} t\right)^{n} L_{n}^{\mu-1}\left(-\frac{z^{2}}{4 a^{2} t}\right), \\
S_{2 n, \nu}^{(2)}(z, t)=\left(4 a^{2} t\right)^{n}\left(\frac{z^{2}}{4 a^{2} t}\right)^{1-\mu} \Phi\left(1-\mu-n, 2-\mu ;-\frac{z^{2}}{4 a^{2} t}\right) \\
=\frac{n ! \Gamma(\mu)}{\Gamma(\mu+n)}\left(4 a^{2} t\right)^{n}\left(\frac{z^{2}}{4 a^{2} t}\right)^{1-\mu} L_{n}^{\mu-1}\left(-\frac{z^{2}}{4 a^{2} t}\right),
\end{gathered}
$$

where $\mu=\frac{\nu+1}{2}$. It should be noted that this formula is valid for $\mu>0$ only.

Using the integral representation for the degenerate hypergeometric function

$$
\begin{aligned}
& \Phi\left(-\frac{\beta}{2}, \mu,-z^{2}\right)=\frac{2 \Gamma(\mu)}{\Gamma(\mu+\beta / 2)} \exp \left(-z^{2}\right) z^{-\mu+1} \\
& \quad \times \int_{0}^{\infty} \exp \left(-x^{2}\right) x^{\mu+\beta} I_{\mu+1}(2 z x) d x
\end{aligned}
$$

and the asymptotic formula

$$
\lim _{z \rightarrow \infty} \frac{e^{-z} I_{\nu}(z)}{\sqrt{2 \pi z}}=1
$$

it is possible to show that

$$
\lim _{z \rightarrow \infty} \frac{1}{z^{\beta}} \Phi\left(-\frac{\beta}{2}, \mu ;-z^{2}\right)=\frac{\Gamma(\mu)}{\Gamma\left(\mu+\frac{\beta}{2}\right)} .
$$

In particular,

$$
\lim _{z \rightarrow \infty} \frac{1}{z^{\beta}} \Phi\left(-\frac{\beta}{2}, 1 ;-z^{2}\right)=\frac{1}{\Gamma\left(1+\frac{\beta}{2}\right)} .
$$

For $\nu=1$, both functions (1) coincide:

$$
S_{\beta, 1}^{(1)}(z, t)=S_{\beta, 1}^{(2)}(z, t)=(2 a \sqrt{t})^{\beta} \Phi\left(-\frac{\beta}{2}, 1 ;-\frac{z^{2}}{4 a^{2} t}\right) .
$$

In this case, the second linearly independent solution of the equation (3) is ([4]) 


$$
\phi_{2}(x)=\Phi\left(-\frac{\beta}{2}, 1 ; x\right) \ln x+\sum_{k=1}^{\infty} M_{k} x^{k},
$$

where

$$
M_{k}=\left(\begin{array}{c}
k \\
-\frac{\beta}{2}
\end{array}\right) \frac{1}{k !} \sum_{m=0}^{k-1}\left(\frac{1}{m-\beta / 2}+\frac{2}{m+1}\right) .
$$

This work is a continuation of the article [11] where we considered problems for generalized heat conduction, and here we consider the case with $\nu=1$ for cylindrical equation.

\section{Problem Definition}

We consider the following problem of heat transfer in solid phase $0<r<\alpha(t)$ and in liquid phase $\alpha(t)<r<\infty$ which can be modelled with cylindrical heat equation

$$
\begin{array}{ll}
\frac{\partial \theta_{1}}{\partial t}=a_{1}^{2}\left(\frac{\partial^{2} \theta_{1}}{\partial r^{2}}+\frac{1}{r} \frac{\partial \theta_{1}}{\partial r}\right), \quad 0<r<\alpha(t), \quad t>0, \\
\frac{\partial \theta_{2}}{\partial t}=a_{2}^{2}\left(\frac{\partial^{2} \theta_{2}}{\partial r^{2}}+\frac{1}{r} \frac{\partial \theta_{2}}{\partial r}\right), \quad \alpha(t)<r<\infty, \quad t>0
\end{array}
$$

with initial conditions

$$
\begin{gathered}
\theta_{1}(0,0)=0, \quad \alpha(0)=0, \\
\theta_{2}(r, 0)=f(r)
\end{gathered}
$$

and boundary conditions

$$
\theta_{1}(\alpha(t), t)=\theta_{2}(\alpha(t), t)=\theta_{m} .
$$

Stefan's condition at free boundary is

$$
-\lambda_{1} \frac{\partial \theta_{1}(\alpha(t), t)}{\partial r}=-\lambda_{2} \frac{\partial \theta_{2}(\alpha(t), t)}{\partial r}+L \gamma \frac{d \alpha}{d t}
$$

and the condition at infinity is

$$
\theta_{2}(\infty, t)=0 .
$$




\section{Problem Solution}

For $\beta=2 n$ we can represent solution of (11)-(17) as form of linear combinations of special functions

$$
\begin{aligned}
& \theta_{1}(r, t)=\sum_{n=0}^{\infty} A_{n}\left(4 a_{1}^{2} t\right)^{n} L_{n}\left(-\frac{r^{2}}{4 a_{1}^{2} t}\right) \\
& +\sum_{n=0}^{\infty} B_{n}\left(4 a_{1}^{2} t\right)^{n}\left[\Phi\left(-n, 1 ;-\frac{r^{2}}{4 a_{1}^{2} t}\right) \ln \left(\frac{r^{2}}{4 a_{1}^{2} t}\right)\right. \\
& \left.+\sum_{k=1}^{\infty} M_{k}\left(-\frac{r^{2}}{4 a_{1}^{2} t}\right)^{k}\right], \\
& \theta_{2}(r, t)=\sum_{n=0}^{\infty} C_{n}\left(4 a_{2} t\right)^{n} L_{n}\left(-\frac{r^{2}}{4 a_{2}^{2} t}\right),
\end{aligned}
$$

where $M_{k}=\left(\begin{array}{l}k \\ n\end{array}\right) \frac{1}{k !} \sum_{m=0}^{k-1}\left(\frac{1}{m+n}+\frac{2}{m+1}\right)$ and $\theta_{1}(r, t), \theta_{2}(r, t)$ are temperatures in solid and liquid zones, $\theta_{m}$ is melting temperature and $A_{n}, B_{n}, C_{n}$ are unknowns which have to be determined. The equations (18) and (19) satisfy the problem (11)-(17). Consider that the function $f(r)=\sum_{n=0}^{\infty} \frac{f^{(n)}(0)}{(n) !} r^{n}$ and free boundary $\alpha(t)=\sum_{n=1}^{\infty} \alpha_{n} t^{n / 2}$ are given. From (14) we have

$$
\sum_{n=0}^{\infty} C_{n} \frac{r^{2 n}}{n !}=\sum_{n=0}^{\infty} \frac{f^{(n)}(0)}{n !} r^{n}
$$

and comparing degree of $r$, we obtain definition for coefficient $C_{n}$ as follows

$$
C_{n}=\frac{f^{(2 n)}(0)}{(2 n) !} n !, \quad n=0,1,2, \ldots
$$

The algorithm to find coefficients $A_{n}$ and $B_{n}$ is that from condition (16) we express coefficients $A_{n}$ and by making substitution to (16) we can get coefficients $B_{n}$. At first, we take $m$-th derivative of (14) and (15) when $\tau=0$, where $\tau=\sqrt{t}$ :

$$
\left.\frac{\partial^{m} \theta_{1}(\alpha(\tau), \tau)}{\partial \tau^{m}}\right|_{\tau=0}=\left.\frac{\partial^{m} \theta_{2}(\alpha(\tau), \tau)}{\partial \tau^{m}}\right|_{\tau=0}=\frac{\partial^{m} \theta_{m}}{\partial \tau^{m}}
$$




$$
\begin{aligned}
& -\left.\lambda_{1} \frac{\partial^{m}}{\partial \tau^{m}}\left[\frac{\partial \theta_{1}(\alpha(\tau), \tau)}{\partial r}\right]\right|_{\tau=0} 0 \\
& =-\left.\lambda_{2} \frac{\partial^{m}}{\partial \tau^{m}}\left[\frac{\partial \theta_{2}(\alpha(\tau), \tau)}{\partial r}\right]\right|_{\tau=0}+L \gamma \frac{d^{m} \alpha}{d \tau^{m}} .
\end{aligned}
$$

We use the Leibniz rule for (22) and (23), then we have

$$
\left.\frac{\partial^{m}\left[\left(4 a_{1}^{2}\right)^{n} \tau^{2} L_{n}(-\delta(\tau))\right]}{\partial \tau^{m}}\right|_{\tau=0}=\left.\frac{\left(4 a_{1}^{2}\right)^{n} m !}{(m-2 n) !}\left[L_{n}(-\delta(\tau))\right]^{(m-2 n)}\right|_{\tau=0}
$$

and

$$
\begin{aligned}
& \left.\frac{\partial^{m}\left[\left(4 a_{1}^{2}\right)^{n} \tau^{n}\left(\Phi[-n, 1 ;-\delta(\tau)] \ln (\delta(\tau))+\sum_{k=1}^{\infty} M_{k}(-\delta(\tau))^{k}\right)\right]}{\partial \tau^{m}}\right|_{\tau=0} \\
& =\left.\frac{\left(4 a_{1}^{2}\right) m !}{(m-2 n) !}\left[\Phi[-n, 1 ;-\delta(\tau)] \ln (\delta(\tau))+\sum_{k=1}^{\infty} M_{k}(-\delta(\tau))^{k}\right]^{(m-2 n)}\right|_{\tau=0} \\
& =\frac{\left(4 a_{1}^{2}\right) m !}{(m-2 n) !} \sum_{l=0}^{m-2 n}\left(\begin{array}{c}
m-2 n \\
l
\end{array}\right)[\Phi[-n, 1 ;-\delta(\tau)]]^{(l)}[\ln (\delta(\tau))]^{(m-2 n-l)} \\
& +\left.\sum_{k=1}^{\infty} M_{k}\left[(-\delta(\tau))^{k}\right]^{(m-2 n)}\right|_{\tau=0},
\end{aligned}
$$

where

$$
\delta(\tau)=\frac{1}{4 a_{1}^{2}}\left(\alpha_{1}+\alpha_{2} \tau+\alpha_{3} \tau^{2}+\ldots\right)^{2}=\frac{1}{4 a_{1}^{2}}\left(\sum_{n=1}^{\infty} \alpha_{n} \tau^{n-1}\right)^{2}, i=1,2 .
$$

Then we use Faa-di Bruno formula for taking derivative from composite function and we get the following results

$$
\begin{aligned}
& \left.\frac{\partial^{m-2 n}\left[L_{n}(-\delta(\tau))\right]}{\partial \tau^{m-2 n}}\right|_{\tau=0} \\
= & \sum_{l=0}^{m-2 n}\left[L_{n}\left(-\delta_{1}\right)\right]^{(l)} \sum_{b_{i}} \frac{(m-2 n) ! \delta_{2}^{b_{2}} \delta_{3}^{b_{3}} \ldots \delta_{m-2 n-l+2}^{b_{m-2 n-l+2}}}{b_{2} ! b_{3} ! \ldots b_{m-2 n-l+2} !}, \\
& \left.\frac{\partial^{m-2 n}[\Phi(-n, 1 ;-\delta(\tau))]}{\partial \tau^{m-2 n}}\right|_{\tau=0} \\
= & \sum_{l=0}^{m-2 n}\left[\Phi\left(-n, 1 ;-\delta_{1}\right)\right]^{(l)} \sum_{b_{i}} \frac{(m-2 n) ! \delta_{2}^{b_{2}} \delta_{3}^{b_{3}} \ldots \delta_{m-2 n-l+2}^{b_{m-2 n-l+2}}}{b_{2} ! b_{3} ! \ldots b_{m-2 n-l+2} !}
\end{aligned}
$$




$$
\begin{gathered}
\left.\frac{\partial^{m-2 n-l}[\ln (\delta(\tau))]}{\partial \tau^{m-2 n-l}}\right|_{\tau=0} \\
=\sum_{p=0}^{m-2 n-l}\left[\ln \left(\delta_{1}\right)\right]^{(p)} \sum_{b_{i}} \frac{(m-2 n-l) ! \delta_{2}^{b_{2}} \delta_{3}^{b_{3}} \ldots \delta_{m-2 n-l-p+2}^{b_{m-2 n-l-p+2}}}{b_{2} ! b_{3} ! \ldots b_{m-2 n-l-p+2} !} \\
\left.\quad \frac{\partial^{m-2 n}\left[(-\delta(\tau))^{k}\right]}{\partial \tau^{m-2 n}}\right|_{\tau=0} \\
=\sum_{l=0}^{m-2 n}\left(-\delta_{1}\right)^{k-l} \sum_{b_{i}} \frac{(m-2 n) ! \delta_{2}^{b_{2}} \delta_{3}^{b_{3}} \ldots \delta_{m-2 n-l+2}^{b_{m-2 n-l+2}}}{b_{2} ! b_{3} ! \ldots b_{m-2 n-l+2} !}
\end{gathered}
$$

where $\delta_{1}=\frac{\alpha_{1}^{2}}{4 a_{i}^{2}}, \quad \delta_{2}=\frac{\alpha_{2}^{2}}{4 a_{i}^{2}}, \ldots, \delta_{m-2 n-l-p+2}=\frac{\alpha_{m-2 n-l-p+2}^{2}}{4 a_{i}^{2}}, i=1,2$ and $b_{1}, b_{2}, b_{3}, \ldots$ satisfy the following equations

$$
\begin{gathered}
b_{2}+b_{3}+\ldots+b_{m-2 n-l-p+2}=m, \\
2 b_{2}+3 b_{3}+\ldots+(m-2 n-l-p+2) b_{m-2 n-l-p+2}=m-2 n .
\end{gathered}
$$

In particular, when $m=0$ and $\tau=0$ we have the following initial coefficients

$$
\begin{gathered}
C_{0}=\theta_{m}, \quad B_{0}=-\frac{L \gamma \alpha_{1}}{\lambda_{1}\left[\frac{4}{\alpha_{0}}+4 a_{1} \sum_{k=1}^{\infty} M_{k} k\left(-\frac{\alpha_{1}}{2 a_{1}}\right)^{2 k-1}\right]}, \\
A_{0}=\theta_{m}+\frac{L \gamma \alpha_{1}\left[\ln \left(\frac{\alpha_{1}^{2}}{4 a_{1}^{2}}\right)+\sum_{k=1}^{\infty} M_{k}\left(-\frac{\alpha_{1}}{2 a_{1}}\right)^{2 k}\right]}{\lambda_{1}\left[\frac{4}{\alpha_{1}}+4 a_{1} \sum_{k=1}^{\infty} M_{k} k\left(-\frac{\alpha_{1}}{2 a_{1}}\right)^{2 k-1}\right]} .
\end{gathered}
$$

By using formulas (24)-(27) we have the following recurrent expressions for conditions (15) and (16)

$$
\begin{aligned}
& \sum_{n=0}^{m} A_{n}\left(4 a_{1}^{2}\right)^{n} \frac{m !}{(m-2 n) !} \sum_{l=0}^{m-2 n}\left[L_{n}\left(-\delta_{1}\right)\right]^{(l)} \\
& \times \sum_{b_{i}} \frac{(m-2 n) ! \delta_{2}^{b_{2}} \delta_{3}^{b_{3}} \ldots \delta_{m-2 n-l+2}^{b_{m-2 n-l+2}}}{b_{2} ! b_{3} ! \ldots b_{m-2 n-l+2} !}
\end{aligned}
$$




$$
\begin{aligned}
& +\sum_{n=0}^{m} B_{n}\left(4 a_{1}^{2}\right)^{n} \frac{m !}{(m-2 n) !} \sum_{l=0}^{m-2 n}\left(\begin{array}{c}
m-2 n \\
l
\end{array}\right)\left[\Phi\left(-n, 1 ;-\delta_{1}\right)\right]^{(l)} \\
& \times \sum_{b_{i}} \frac{(m-2 n) ! \delta_{2}^{b_{2}} \delta_{3}^{b_{3}} \ldots \delta_{m-2 n-l+2}^{b_{m-2 n-l+2}}}{b_{2} ! b_{3} ! \ldots b_{m-2 n-l+2} !} \\
& \times \sum_{p=0}^{m-2 n-l}\left[\ln \left(\delta_{1}\right)\right]^{(p)} \sum_{b_{i}} \frac{(m-2 n-l) ! \delta_{2}^{b_{2}} \delta_{3}^{b_{3}} \ldots \delta_{m-2 n-l-p+2}^{b_{m-2 n-l-p+2}}}{b_{2} ! b_{3} ! \ldots b_{m-2 n-l-p+2} !} \\
& +\sum_{k=1}^{\infty} M_{k} \sum_{l=0}^{m-2 n} \delta_{1}^{k-l} \sum_{b_{i}} \frac{(m-2 n) ! \delta_{2}^{b_{2}} \delta_{3}^{b_{3}} \ldots \delta_{m-2 n-l+2}^{b_{m-2 n-l+2}}}{b_{2} ! b_{3} ! \ldots b_{m-2 n-l+2} !}=0
\end{aligned}
$$

and

$$
\begin{gathered}
-\lambda_{1}\left[\sum_{n=0}^{m} A_{n}\left(4 a_{1}^{2}\right)^{n} \frac{m !}{(m-2 n) !} \sum_{l=0}^{m-2 n}\left[\frac{\partial}{\partial r} L_{n}\left(-\delta_{1}\right)\right]^{(l)}\right. \\
\times \sum_{b_{i}} \frac{(m-2 n) ! \delta_{2}^{b_{2}} \delta_{3}^{b_{3}} \ldots \delta_{m-2 n-l+2}^{b_{m-2 n-2}}}{b_{2} b_{3} ! \ldots b_{m-2 n-l+2} !}
\end{gathered}
$$

$$
\begin{aligned}
& +\sum_{n=0}^{m} B_{n}\left(4 a_{1}^{2}\right)^{2} \frac{m !}{(m-2 n) !} \sum_{l=0}^{m-2 n}\left(\begin{array}{c}
m-2 n \\
l
\end{array}\right)\left[\frac{\partial}{\partial r} \Phi\left(-n, 1 ;-\delta_{1}\right)\right]^{(l)} \\
& \times \sum_{b_{i}} \frac{(m-2 n) ! \delta_{2}^{b_{2}} \delta_{3}^{b_{3}} \ldots \delta_{m-2 n-l+2}^{b_{m-2 n-2}}}{b_{2} b_{3} ! \ldots b_{m-2 n-l+2} !} \\
& \times \sum_{p=0}^{m-2 n-l}\left[\ln \left(\delta_{1}\right)\right]^{(p)} \sum_{b_{i}} \frac{(m-2 n-l) ! \delta_{2}^{b_{2}} \delta_{3}^{b_{3}} \ldots \delta_{m-2 n-l-p+2}^{b_{m-2 n-l-2}}}{b_{2} b_{3} ! \ldots b_{m-2 n-l-p+2} !} \\
& +\sum_{l=0}^{m-2 n}\left(\begin{array}{c}
m-2 n \\
l
\end{array}\right)\left[\Phi\left(-n, 1 ;-\delta_{1}\right)\right]^{(l)} \sum_{b_{i}} \frac{(m-2 n) ! \delta_{2}^{b_{2}} \delta_{3}^{b_{3}} \ldots \delta_{m-2 n-l+2}^{b_{m-2 n-l+2}}}{b_{2} b_{3} ! \ldots b_{m-2 n-l+2} !}
\end{aligned}
$$




$$
\begin{aligned}
& \times \sum_{p=0}^{m-2 n-l}(-1)^{p} \frac{1}{\delta_{1}^{p+1}} \sum_{b_{i}} \frac{(m-2 n-l) ! \delta_{2}^{b_{2}} \delta_{3}^{b_{3}} \ldots \delta_{m-2 n-l-p+2}^{b_{m-2 n-l-p+2}}}{b_{2} b_{3} ! \ldots b_{m-2 n-l-p+2} !} \\
& +\sum_{k=1}^{\infty} M_{k} \sum_{l=0}^{m-2 n}\left(\begin{array}{c}
m-2 n \\
l
\end{array}\right) \delta_{1}^{k-l} \sum_{b_{i}} \frac{(m-2 n) ! \delta_{2}^{b_{2}} \delta_{3}^{b_{3}} \ldots \delta_{m-2 n-l+2}^{b_{m-2 n-l+2}}}{b_{2} b_{3} ! \ldots b_{m-2 n-l+2} !} \\
& \left.\times \sum_{p=0}^{m-2 n-l} \beta_{1}^{(p)} \sum_{b_{i}} \frac{(m-2 n-l) ! \delta_{2}^{b_{2}} \delta_{3}^{b_{3}} \ldots \delta_{m-2 n-l-p+2}^{b_{m-2 n-l-p+2}}}{b_{2} b_{3} ! \ldots b_{m-2 n-l-p+2} !}\right] \\
& =-\lambda_{2}\left[\sum_{n=0}^{m} C_{n}\left(4 a_{2}^{2}\right) n \frac{m !}{(m-2 n) !} \sum_{l=0}^{m-2 n}\left[\frac{\partial}{\partial r} L_{n}\left(-\delta_{1}\right)\right]^{(l)}\right. \\
& \left.\times \sum_{b_{i}} \frac{(m-2 n) ! \beta_{2}^{b_{2}} \beta_{3}^{b_{3}} \ldots \beta_{m-2 n-l+2}^{b_{m-2 n-l+2}}}{b_{2} b_{3} ! \ldots b_{m-2 n-l+2} !}\right]+L \gamma m ! \alpha_{m}
\end{aligned}
$$

where $\beta(\tau)=\frac{1}{2 a_{1}^{2}}\left(\sum_{n=1}^{\infty} \alpha_{n} \tau^{n-1}\right)^{2}$. From recurrent expression (29) we express coefficient $A_{n}$. Making substitutions to (30), we can determine $B_{n}$ as free boundary is given and coefficient $C_{n}$ can be found from (21).

\section{Convergence of Series}

The convergence of series (18)-(19) can be proved as follows. Let $\alpha\left(t_{0}\right)=\eta_{0}$ for any $t=t_{0}$. Then the series (18) can be written as

$$
\begin{aligned}
& \theta_{1}\left(r, t_{0}\right)=\sum_{n=0}^{\infty} A_{n}\left(4 a_{1}^{2} t_{0}\right) L_{n}\left(-\frac{r_{0}^{2}}{4 a_{1}^{2} t_{0}}\right)+\sum_{n=0}^{\infty} B_{n}\left(4 a_{1}^{2} t_{0}\right)^{n} \\
& \times\left[\Phi\left(-n, 1 ;-\frac{r_{0}^{2}}{4 a_{1}^{2} t_{0}}\right) \ln \left(\frac{r_{0}^{2}}{4 a_{1}^{2} t_{0}}\right)+\sum_{k=1}^{\infty} M_{k}\left(-\frac{r_{0}^{2}}{4 a_{1}^{2} t_{0}}\right)^{k}\right] .
\end{aligned}
$$

The series (18) and (19) must be convergence because $\theta_{1}(\alpha(t), t)=\theta_{2}(\alpha(t), t)=\theta_{m}$. Then there exists some constant $D_{1}$ independent of $n$ and for the first term of (31) we have

$$
\left|A_{n}\right|<D_{1} /\left(4 a_{1}^{2} t_{0}\right)^{n} L_{n}\left(-\frac{\eta_{0}^{2}}{4 a_{1}^{2} t_{0}}\right)
$$

Since $A_{n}$ bounded, then multiply both sides of (32) by 


$$
\begin{aligned}
& \left(4 a_{1}^{2} t\right)^{n} L_{n}\left(-\frac{(\alpha(t))^{2}}{4 a_{1}^{2} t}\right) \text { we obtain } \\
& \qquad \sum_{n=0}^{\infty} A_{n}\left(4 a_{1}^{2} t\right)^{n} L_{n}\left(-\frac{(\alpha(t))^{2}}{4 a_{1}^{2} t}\right) \\
& \quad<D_{1} \sum_{n=0}^{\infty} \frac{\left(4 a_{1}^{2} t\right)^{n} L_{n}\left(-\frac{(\alpha(t))^{2}}{4 a_{1}^{2} t}\right)}{\left(4 a_{1}^{2} t_{0}\right)^{n} L_{n}\left(-\frac{\eta^{2}}{4 a_{1}^{2} t_{0}}\right)}<D_{1} \sum_{n=0}^{\infty}\left(\frac{t}{t_{0}}\right)^{n} .
\end{aligned}
$$

For the second term of (33) we consider that there exists some constant $D_{2}$ and we obtain

$$
\begin{aligned}
& \left|B_{n}\right|>D_{2} /\left\{( 4 a _ { 1 } ^ { 2 } t _ { 0 } ) ^ { n } \left[\left(\Phi-n, 1 ;-\frac{r_{0}^{2}}{4 a_{1}^{2} t_{0}}\right) \ln \left(\frac{r_{0}^{2}}{4 a_{1}^{2} t_{0}}\right)\right.\right. \\
& \left.\left.+\sum_{k=1}^{\infty} M_{k}\left(-\frac{r_{0}^{2}}{4 a_{1}^{2} t_{0}}\right)^{k}\right]\right\} .
\end{aligned}
$$

As $B_{n}$ is bounded, multiplying both sides of (34) by

$$
\left(4 a_{1}^{2} t\right)^{n}\left[\Phi\left(-n, 1 ;-\frac{(\alpha(t))^{2}}{4 a_{1}^{2} t}\right) \ln \left(\frac{(\alpha(t))^{2}}{4 a_{1}^{2} t}\right)+\sum_{k=1}^{\infty} M_{k}\left(-\frac{(\alpha(t))^{2}}{4 a_{1}^{2} t}\right)^{k}\right],
$$

we get

$$
\begin{aligned}
& B_{n}\left(4 a_{1}^{2} t\right)^{n}\left[\Phi\left(-n, 1 ;-\frac{(\alpha(t))^{2}}{4 a_{1}^{2} t}\right) \ln \left(\frac{(\alpha(t))^{2}}{4 a_{1}^{2} t}\right)+\sum_{k=1}^{\infty} M_{k}\left(-\frac{(\alpha(t))^{2}}{4 a_{1}^{2} t}\right)^{k}\right] \\
& <D_{2} \frac{\left(4 a_{1}^{2} t\right)^{n} \Phi\left[\left(-n, 1 ;-\frac{(\alpha(t))^{2}}{4 a_{1}^{2} t}\right) \ln \left(\frac{(\alpha(t))^{2}}{4 a_{1}^{2} t}\right)+\sum_{k=1}^{\infty} M_{k}\left(-\frac{(\alpha(t))^{2}}{4 a_{1}^{2} t}\right)^{k}\right]}{\left(4 a_{1}^{2} t_{0}\right)^{n}\left[\Phi\left(-n, 1 ;-\frac{\eta^{2}}{4 a_{1}^{2} t_{0}}\right) \ln \left(\frac{\eta^{2}}{4 a_{1}^{2} t_{0}}\right)+\sum_{k=1}^{\infty} M_{k}\left(-\frac{\eta^{2}}{4 a_{1}^{2} t_{0}}\right)^{k}\right]} \\
& <D_{2} \sum_{n=0}^{\infty}\left(\frac{t}{t_{0}}\right)^{n} .
\end{aligned}
$$

These geometric series and $\theta_{1}$ converge for all $r<\mu_{0}$ and the same is the $\theta_{2}$ convergence for all $r>\mu_{0}$ and $t<t_{0}$. The convergence for equation (19) and $\alpha(t)$ can be determined analogously. 


\section{Conclusion}

The undetermined coefficients of heat in liquid and solid phases are found from (14) by comparing degree of cylindrical material radius in expression (20) and from recurrent formulas (29) and (30) for the conditions (15) and (16) which obtained by using Leibniz Rule and Faa di Bruno formulas.

\section{Acknowledgment}

The research is supported by the Grant Project AP09258948 from the Ministry of Science and Education of the Republic of Kazakhstan.

\section{References}

[1] M. Jenaliyev, M. Amangaliyeva, M. Kosmakova and M. Ramazanov, About Dirichlet boundary value problem for the heat equation in the infinite angular domain, Bound. Value Probl., 2014 (2014), Art. No 213, 1-21.

[2] V. Zvyagin and V. Orlov, On the weak solvability of one system of thermoviscoelasticity, In: AIP Conf. Proc., 1626 (Internat. Conf. on Advancements in Mathematical Sciences (AMS), Antalya, Turkey, Nov. 05-07, 2015), Ed. by: A. Ashyralyev et al., Amer. Inst. Phys. (2015).

[3] D. Slota, Homotopy perturbation method for solving the two-phase inverse Stefan problem, Numer. Heat Transfer. Part A: Appl, 59, No 10 (2011), 755-768.

[4] G. Szego, Orthogonal Polynomials, Amer. Math. Soc., Providence, RI (1939).

[5] G-Q. Chen, H. Shahgholian and J-L. Vazquez, Free boundary problems: the forefront of current and future developments, Phil. Trans. R. Soc. (2015); A373:20140285-1-20140285-8.

[6] A. Friedman, Free boundary problems in biology, Phil. Trans. R. Soc. (2015); \# A373: 20140368-1-20140368-16.

[7] A. Friedman, Free boundary problems for parabolic equations: I. Melting of solids, J. Math. Mec., 8 (1959), 499-517. 
[8] A. Bermidez and M.V. Otero, An existence result for a two-phase Stefan problem arising in metal casting, Math. Meth. Appl. Sci., 29 (2006), 325350.

[9] L.I. Rubinstein, The Stefan Problem, Vol. 27 of Trans. Math. Monogr., AMS, Providence (1971).

[10] P.C. Rosenbloom and D.V. Widder, Expansions in terms of heat polynomials and associated functions, Trans. Amer. Math. Soc., 92 (1959), 220-266.

[11] S.N. Kharin and T.A. Nauryz, Two-phase Stefan problem for generalized heat equation, News of the National Academy of Sciences of the Republic of Kazakhstan, Physico-Mathematical Ser., 330, Vol. 2 (2020), 40-49. 